\title{
Mitochondrial PIP3-binding protein FUNDC2 supports platelet survival via AKT signaling pathway
}

\author{
Qi Ma ${ }^{1,2} \cdot$ Chongzhuo Zhu ${ }^{1} \cdot$ Weilin Zhang ${ }^{1} \cdot \mathrm{Na} \mathrm{Ta}^{1} \cdot$ Rong Zhang ${ }^{1} \cdot$ Lei Liu ${ }^{1} \cdot$ Du Feng ${ }^{3} \cdot$ Heping Cheng $^{2} \cdot$ \\ Junling Liu ${ }^{4}$ Quan Chen ${ }^{1}$
}

Received: 1 November 2017 / Revised: 21 March 2018 / Accepted: 19 April 2018

๑) ADMC Associazione Differenziamento e Morte Cellulare 2018

\begin{abstract}
Platelets undergo apoptosis in response to a variety of stimuli in the circulation. Mitochondria in platelets are essential for their apoptosis. Specifically, pro-survival protein $B C L-x_{L}$ on mitochondria is the key regulator of platelet lifespan. Here we identify an outer mitochondrial membrane protein FUNDC2 for platelet survival. FUNDC2 knockout mice carrying excessively apoptotic platelets exhibit thrombocytopenia in response to hypoxia. Mechanistically, FUNDC2 binds the lipid PIP3 via its unique, highly conserved N-terminal motif. FUNDC2 deficiency abrogates the phosphorylation of AKT and its substrate BAD in a PIP3/PI3K-dependent manner, which suppresses BCL- $\mathrm{x}_{\mathrm{L}}$. Indeed, FUNDC2 deficiency shortens the platelet lifespan under stress. Thus, this FUNDC2/AKT/BCL- $\mathrm{x}_{\mathrm{L}}$ axis signifies a balance between platelet survival and apoptosis at the single organelle level and provides new insight for platelet-related diseases as well.
\end{abstract}

\section{Introduction}

Platelets are small cytoplasmic fragments that contain mitochondria but no nucleus [1]. They are exposed to a diversity of stresses, such as shear force, high-oxygen, oxidative damage, and hypoxia, during their circulation in peripheral blood [2-6]. A number of studies have suggested that platelets can undergo apoptosis in response to various stimuli [7-11]. The high turnover rate of platelets, with a lifespan around 5 days in mouse or 10 days in humans [12], also requires accurate modulation of intrinsic programmed cell death [13]. Mitochondria are essential for platelet apoptosis. They are not only the powerhouse in cells to

\section{Edited by L Scorrano}

Electronic supplementary material The online version of this article (https://doi.org/10.1038/s41418-018-0121-8) contains supplementary material, which is available to authorized users.

Qi Ma

maq@pku.edu.cn

1 State Key Laboratory of Membrane Biology, Institute of Zoology, Chinese Academy of Sciences, Beijing, China

2 Beijing Key Laboratory of Cardiometabolic Molecular Medicine, Peking-Tsinghua Center for Life Sciences, Institute of Molecular Medicine, Peking University, Beijing, China provide energy but also contain key regulators of cell death. It has been shown that the pro-survival protein $\mathrm{BCL}-\mathrm{x}_{\mathrm{L}}$, which is a member of the extended B-cell lymphoma-2 (BCL-2) protein family on mitochondria [14], is the key mediator of platelet lifespan $[15,16]$. Genetic ablation of BCL- $\mathrm{x}_{\mathrm{L}}$ results in reduced platelet half-life and marked thrombocytopenia in mice. Pharmacologic inhibition of BCL- $\mathrm{x}_{\mathrm{L}}$ by ABT737, the pro-apoptotic BCL-2 homology 3 (BH3) mimetic [17], also leads to dose-dependent thrombocytopenia $[15,18]$.

The serine/threonine protein kinase AKT (also known as protein kinase $\mathrm{B}, \mathrm{PKB}$ ) is a central node in the regulation of cell fate $[19,20]$. AKT is evoked at the cell membrane by binding to the lipid phosphatidylinositol-3,4,5-trisphosphate (PIP3) which is an important second messenger in mammalian cells [21]. Upon stimulation, AKT can translocate into the cytosol and the nucleus, as well as the mitochondria [22], and phosphorylate a variety of substrates to promote

3 Guangdong Key Laboratory of Age-related Cardiac-cerebral Vascular Disease, Department of Neurology, Institute of Neurology, The Affiliated Hospital of Guangdong Medical University, Guangdong Medical University, Zhanjiang, China

4 Shanghai Key Laboratory of Tumor Microenvironment and Inflammation, Department of Biochemistry and Molecular Cell Biology, School of Medicine, Shanghai Jiao Tong University, Shanghai, China 
cell survival. However, whether and how AKT regulates platelet survival are still largely unknown.

In this study, we identified a novel mitochondrial outer membrane protein FUNDC2 that binds PIP3 directly. FUNDC2 deficiency decreased PIP3 level and abrogated the phosphorylation of AKT and BAD. Eventually, BCL$\mathrm{x}_{\mathrm{L}}$ was inhibited by unphosphorylated BAD. Thus, FUNDC2-deficient mice displayed thrombocytopenia in response to hypoxia. The newly characterized FUNDC2/ AKT/BCL- $x_{L}$ signaling pathway protects platelets against apoptotic stress.

\section{Methods}

\section{Mice}

All protocols were approved by the Institutional Animal Care and Use Committee of the Institute of Zoology accredited by AAALAC International. Adult (3-5-monthaged) male animals were used in this study. For the generation of FUNDC2-null mice, the FUNDC2-targeting vector consisted of 2 genomic fragments and the neomycin resistance gene $(\mathrm{NeO})$, which replaced the second coding exon of the FUNDC2 gene following homologous recombination. Embryonic stem cell clones (C57BL/ 6 strain) were used to generate chimeric mice and eventually FUNDC2-null mice. For hypoxia treatment, mice were maintained in $\mathrm{O}_{2}$-controlled chambers $\left(\begin{array}{rl}\sim \% & \mathrm{O}_{2}\end{array}\right)$ with a 12-h light/dark cycle and were given free access to food and water. For bone marrow transplantation, recipient mice were X-ray-irradiated at a single dose of 8.5 Gy.

\section{Antibodies, reagents, and plasmids}

FUNDC2 antibody (Ab) was generated by immunizing rabbits with recombinant FUNDC2 protein and affinity purified by our laboratory. Phospho-Akt (Ser473) mouse $\mathrm{mAb}$ (\#4051) and phospho-Bad (Ser136) rabbit mAb (\#4366) were purchased from Cell Signaling. In total, $1 \mu \mathrm{g} /$ $\mathrm{ml}$ (final concentration) Abs were used for immunoblotting and $10 \mu \mathrm{g} / \mathrm{ml}$ for immunoprecipitation or immunostaining. Unless otherwise noted, all Abs were purchased from Cell Signaling and all chemicals were from Sigma Aldrich. Fulllength $F U N D C 2$ was amplified from a human or mouse cDNA library by PCR. DNA fragments corresponding to full-length $F U N D C 2$ or the indicated mutants were inserted into pCMV-N-HA vector between the EcoR I and Xho I restriction sites or pcDNA4/TO/myc-His B vector between Kpn I and Xho I. For protein expression and purification, indicated sequences were inserted into pGEX-4T-1 vector between BamH I and $E c o R$.

\section{Cell culture and plasmid transfection}

Primary MEFs were obtained from day E13.5 embryos of mice. MEF and HeLa cells were grown in DMEM highglucose medium (Invitrogen) with $10 \% \mathrm{FBS}$ at $37^{\circ} \mathrm{C}$ and $5 \% \quad \mathrm{CO}_{2}$. When cells reached $\sim 60 \%$ confluence, gene transfer was performed by plasmid transfection using Lipofectamine-2000 kits (Invitrogen).

\section{Mitochondrial fractionation}

HeLa cells were collected and resuspended in hypotonic buffer (10 mM Tris, $150 \mathrm{mM}$ EGTA, $200 \mathrm{mM}$ sucrose, $\mathrm{pH}$ 7.4). After gentle homogenization with a Dounce homogenizer, cells were centrifuged at $1000 \times g$ for $10 \mathrm{~min}$ to remove nuclei and intact cells. Mitochondria were pelleted by centrifugation at $10,000 \times g$ for $10 \mathrm{~min}$.

\section{Protease $\mathrm{K}$ digestion assay}

Isolated mitochondria were suspended in hypotonic buffer and incubated on ice with $100 \mu \mathrm{g} / \mathrm{ml}$ proteinase $\mathrm{K}$ containing 1\% TritonX-100 or not for 30 min. Digestion was terminated by adding PMSF with a final concentration of 7 $\mathrm{mM}$ on ice for $5 \mathrm{~min}$. Mitochondrial proteins were immunoblotted.

\section{Immunogold electron microscopy}

Platelets were fixed with $2 \%$ paraformaldehyde at $4{ }^{\circ} \mathrm{C}$ overnight and dehydrated in a graded ethanol series and embedded in acrylic resin (LR White). Ultrathin sections of $70 \mathrm{~nm}$ were mounted on nickel grids. After blocking in PBS containing $1 \%$ BSA, samples were first incubated with primary antibody in $1 \%$ BSA at $4{ }^{\circ} \mathrm{C}$ overnight, washed 5 times for $5 \mathrm{~min}$ in $0.5 \% \mathrm{BSA}$, and labeled with $20 \mathrm{~nm}$ or 10 $\mathrm{nm}$ gold-conjugated particles in $1 \%$ BSA. Later, grids were washed 5 times for $5 \mathrm{~min}$ in $0.5 \%$ BSA. Finally, grids were incubated for $15 \mathrm{~min}$ in $1 \%$ glutaraldehyde, washed twice for $5 \mathrm{~min}$ in PBS then 3 times in distilled water, stained, and dried at room temperature. The samples were visualized using a $120 \mathrm{kV}$ Jeol electron microscope at $80 \mathrm{kV}$ and images were captured using an AMT digital camera.

\section{Immunoblotting, co-immunoprecipitation, and immunostaining}

Tissues or cells were lysed in lysis buffer (30 mM HEPES, $100 \mathrm{mM} \mathrm{NaCl}, 0.5 \%$ Nonidet P (NP)-40, protease inhibitors mixture, $\mathrm{pH} 7.6$ ) on ice for $10 \mathrm{~min}$ and the lysates were centrifuged at 13,000 rpm for $10 \mathrm{~min}$. For immunoblotting, proteins were resolved by SDS-PAGE transferred onto nitrocellulose membranes and detected with the indicated 
antibodies. For co-immunoprecipitation, immunoprecipitation was performed using the indicated antibodies, followed by immunoblotting analysis. For immunostaining, cells were transfected with the indicated plasmids and treated with the corresponding antibodies before incubation with fluorescein isothiocyanate (FITC)-, CY3- or CY5conjugated second antibodies (DAKO). Images were collected with a laser scanning confocal microscope (LSM700, Zeiss).

\section{Protein purification}

The vector pGEX-4T-1 containing the indicated DNA sequence was transfected into $E$. coli strain BL-21(DE3). Cells were grown in Luria-Bertani medium to OD600 0.6 then IPTG $(0.1 \mathrm{mM}$ final concentration) was added to induce the protein expression at $16^{\circ} \mathrm{C}$ overnight. Cells were lysed by sonication and the inclusion bodies were discarded by centrifuging at $12,000 \times g$ for $1 \mathrm{~h}$. GST-fused proteins were purified using glutathione (GSH)-Sepharose 4 Fast Flow beads (Amersham Biosciences).

\section{Protein-lipid overlay assay}

Hydrophobic membranes spotted with 100 pM of indicated lipids (Echelon Biosciences) were purchased and binding assays were performed as recommended by the manufacturer. Briefly, the membranes were blocked with PBST containing 3\% BSA and incubated with recombinant GSTFUNDC2 protein for $1 \mathrm{~h}$ at room temperature. Membranes were then analyzed by SDS-PAGE and immunoblotted with GST antibody.

\section{Pulldown assay}

For protein-lipid pulldown, $1 \mu \mathrm{g}$ GST-fused proteins and 20 $\mu \mathrm{L}$ PIP3-coated beads (Echelon Biosciences) were mixed in $1 \mathrm{ml}$ binding buffer ( $50 \mathrm{mM}$ Tris, $150 \mathrm{mM} \mathrm{NaCl}, 0.05 \%$ $\mathrm{NP}-40, \mathrm{pH} 7.5$ ) and rotated for $15 \mathrm{~min}$ at room temperature. After centrifugation at $13,000 \mathrm{rpm}$ for $10 \mathrm{~min}$, pellets were resuspended in $1 \mathrm{ml}$ binding buffer and washed 3 times. Pellets and flowthrough were analyzed by SDS-PAGE and immunoblotted with GST antibody.

\section{Hematological analysis}

Complete and differential blood cell counts were determined using automated counting techniques (Beckman Coulter).

\section{Thrombopoietin analysis}

Blood was collected from the retroorbital sinus and incubated at room temperature for $2 \mathrm{~h}$. After centrifugation at
$5500 \mathrm{rpm}$ for $20 \mathrm{~min}$, the supernatant was centrifuged again under the same conditions and serum collected. Thrombopoietin levels were measured by enzyme-linked immunosorbent assay (ELISA) using the Quantikine Mouse TPO Immunoassay kit (R\&D Systems) according to the manufacturer's instructions.

\section{Platelet preparation}

Whole blood from mice was mixed with $1 / 10$ volume of White's anticoagulant $(2.94 \%$ sodium citrate, $136 \mathrm{mM}$ glucose, $\mathrm{pH}$ 6.4). Platelet-rich plasma was obtained by centrifugation at $150 \times g$ for $10 \mathrm{~min}$. Washed platelets were obtained by centrifugation at $800 \times g$ for $10 \mathrm{~min}$ and resuspended in modified Tyrode's buffer $(12 \mathrm{mM} \mathrm{NaHCO} 3,138$ $\mathrm{mM} \mathrm{NaCl}, 5.5 \mathrm{mM}$ glucose, $2.9 \mathrm{mM} \mathrm{KCl}, 2 \mathrm{mM} \mathrm{MgCl}_{2}$, $0.42 \mathrm{mM} \mathrm{NaH} \mathrm{PO}_{4}, 10 \mathrm{mM}$ HEPES, pH 7.4). Washed platelets were left to rest for $30 \mathrm{~min}$ before use.

\section{Ex vivo assay}

Rest platelets were resuspended in modified Tyrode's buffer (12 mM NaHCO $3,138 \mathrm{mM} \mathrm{NaCl}, 5.5 \mathrm{mM}$ glucose, $2.9 \mathrm{mM}$ $\mathrm{KCl}, 2 \mathrm{mM} \mathrm{MgCl}_{2}, 0.42 \mathrm{mM} \mathrm{NaH} \mathrm{PO}_{4}, 10 \mathrm{mM}$ HEPES, $\mathrm{pH} 7.4$ ) and were treated in $22{ }^{\circ} \mathrm{C}$ by $8 \% \mathrm{O}_{2}$ for $2-4 \mathrm{~h}$ with $5 \mu \mathrm{M}$ Z-VAD-FMK, or $5 \mu \mathrm{M}$ LY294002, or not.

\section{Flow cytometry analysis}

For PIP3 study, platelets derived from mice were digitoninpermeabilized for $5 \mathrm{~min}$ on ice and labeled with PIP3 antibody/FITC-conjugated second antibody. For apoptosis analysis, platelets were stained with FITC-conjugated annexin $\mathrm{V}$ for quantitative apoptosis assay or tetramethylrhodamine ethyl ester (TMRE, Invitrogen) for mitochondrial membrane potential measurement. For megakaryocytes analysis, bone marrow from femurs was flushed with PBS and dispersed with a transfer pipette. After filtration through a $100 \mu \mathrm{m}$ cell strainer, the cell suspension was centrifuged at $1000 \times g$ for $5 \mathrm{~min}$. Cells were then resuspended in $1 \mathrm{ml}$ PBS containing 5\% FBS. Cells were labeled with phycoerythrin (PE)-conjugated anti-GPIb antibody (Emfret) and FITC-conjugated anti-CD41 antibody on ice for $30 \mathrm{~min}$ before flow cytometry analysis of megakaryocytes.

\section{Platelet clearance assay}

Mice were injected intravenously with $300 \mu \mathrm{g} N$ hydroxysuccinimido (NHS)-biotin (Sigma) in normal saline containing $10 \%$ DMSO. At various time points whole tail blood was isolated and mixed with BSGC buffer (13.6 $\mathrm{mM}$ tri-sodium citrate, $116 \mathrm{mM} \mathrm{NaCl}, 8.6 \mathrm{mM} \mathrm{Na}_{2} \mathrm{HPO}_{4}$, 
$0.9 \mathrm{mM}$ EDTA, $1.6 \mathrm{mM} \mathrm{KH_{2 }} \mathrm{PO} 4,11.1 \mathrm{mM}$ glucose). After two centrifugation steps, the platelet pellet was resuspended in BSS buffer $(149 \mathrm{mM} \mathrm{NaCl}, 2.5 \mathrm{mM} \mathrm{CaCl} 2,1.2 \mathrm{mM}$ $\mathrm{MgSO}_{4}, 3.7 \mathrm{mM} \mathrm{KCl}, 1.2 \mathrm{mM} \mathrm{KH} \mathrm{PO}_{4}, 0.8 \mathrm{mM} \mathrm{K}_{2} \mathrm{HPO}_{4}$, $7.4 \mathrm{mM}$ HEPES, 3\% FBS) and stained with FITCconjugated anti-CD41 antibody (BD) and PE-conjugated streptavidin (BD) for $1 \mathrm{~h}$ on ice. Flow cytometry analysis was performed using FACScan (BD) and all data were analyzed with Cell Quest software (BD).

\section{Adoptive platelet transfer assay}

Mice were injected intravenously with $300 \mu$ g NHS-biotin. $30 \mathrm{~min}$ later, platelets were isolated and resuspended in normal saline before intravenous injection into recipient mice.

\section{Statistical analysis of data}

Statistical data are presented as mean \pm SEM. Significance was calculated by Student's $t$-test, or Mann-Whitney $U$ test. Differences between groups were examined using one-way analysis of variance (ANOVA) or repeated-measures ANOVA. NS no statistical significance; ${ }^{*} p<0.05 ; * * p<$ $0.01 ; * * * p<0.001$. The number of independent experiments or mice was clarified in figure legends or in column of each figure.

\section{Results}

\section{Identification of FUNDC2 as a mitochondrial protein}

In our previous study, we found that mitochondrial protein FUNDC1 mediates mitophagy and regulates platelet functions [23, 24]. FUNDC2 (FUN14 domain-containing 2, also known as HCBP6) $[25,26]$ is a homolog of FUNDC1. FUNDC2 is highly conserved in vertebrates (Fig. 1a). However, its domain structure or biological function was previously uncharacterized. First, we validated its mitochondrial localization. Confocal imaging analysis showed that HA-tagged FUNDC2 (FUNDC2-HA) localized exclusively to mitochondria in mouse fibroblasts (Fig. 1b). Fractionation assays with HeLa cell lysates also biochemically confirmed the localization of endogenous FUNDC2 to mitochondria (Fig. 1c). Three hydrophobic transmembrane (TM) regions in FUNDC2 (supplemental Figure S1a) were predicted using TMpred software. Our topology study demonstrated that both TM1 and TM2 are required for its mitochondrial localization (supplemental Figure S1, b and c). Furthermore, proteinase K digestion assays revealed that FUNDC2 is localized in the outer mitochondrial membrane (OMM) with both its $\mathrm{N}$ - and C-termini facing the cytosol (Fig. 1d,e).

\section{FUNDC2 knockout mice display hypoxia-induced thrombocytopenia}

FUNDC2 mRNA is ubiquitous in a variety of tissues of mice (Fig. 2a). However, expression profiling showed an unexpected high protein level of FUNDC2 in platelets (Fig. 2b). Immunogold electron micrographs also confirmed the enrichment of FUNDC2 on mitochondrial surface in platelets (Fig. 2c). Moreover, its homolog FUNDC1 regulates platelet functions [24]. Thus, we focused on studying the role of FUNDC2 in platelets. To further explore the physiological relevance of FUNDC2, we established FUNDC2 knockout (KO) mice (supplemental FigureS2, a and b). Mice lacking FUNDC2 display gross normality (Fig. 2d and supplemental FigureS3, c-e). Specifically, FUNDC2-deficient mice displayed thrombocytopenia (platelet counts $\sim 700 \times 10^{3} / \mu \mathrm{l}$ ) compared to the wild-type (WT) littermates (platelet counts $\sim 1100 \times 10^{3} / \mu \mathrm{l}$ ) in response to chronic hypoxia, a mild stress, for 5 days (Fig. 2e). Moreover, the thrombopoietin (TPO) levels in FUNDC2-KO or WT mice were little changed during hypoxia treatment (supplemental Figure S3a). In contrast, other peripheral blood cells were unaffected in the loss of FUNDC2 (Table 1), indicating that FUNDC2 deficiency have no effects on hematopoietic stem cells and other lineages of blood cells. Notably, hypoxia even triggered thrombocytopenia in FUNDC2-null mice after the treatment for only 1 day. However, no further decrease of platelet count was observed at the duration (14 days) of the treatment. Moreover, the thrombocytopenia could reverse in 7 days after the animals were brought back to normoxic conditions (Fig. 2f). Platelets circulate for about 5 days in mice, thus this result implied that the platelet generation of FUNDC2 knockout mice remains unimpaired. Additionally, transplantation of bone marrow derived from WT animals rescued hypoxiainduced thrombocytopenia in FUNDC2-deficient mice. In contrast, WT mice transplanted with FUNDC2-KO bone marrow displayed decreased platelet count (Fig. 2g). Collectively, these data suggest that the genetic ablation of FUNDC2 attenuates platelet survival. Platelets are produced from megakaryocytes in the bone marrow and spleen and eventually destroyed by the reticuloendothelial system, primarily in the liver and spleen. Spleens in FUNDC2 mutant mice were morphologically similar to those of WT counterparts (supplemental Figure S3b) and the numbers of mature megakaryocytes with or without FUNDC2 were comparable (supplemental Figure S3, c and d).

\section{FUNDC2 deficiency shortens platelet lifespan in response to hypoxia stress}

To monitor thrombocyte survival in the circulation, platelets were biotin-labeled and tracked in vivo. Loss of FUNDC2 
a
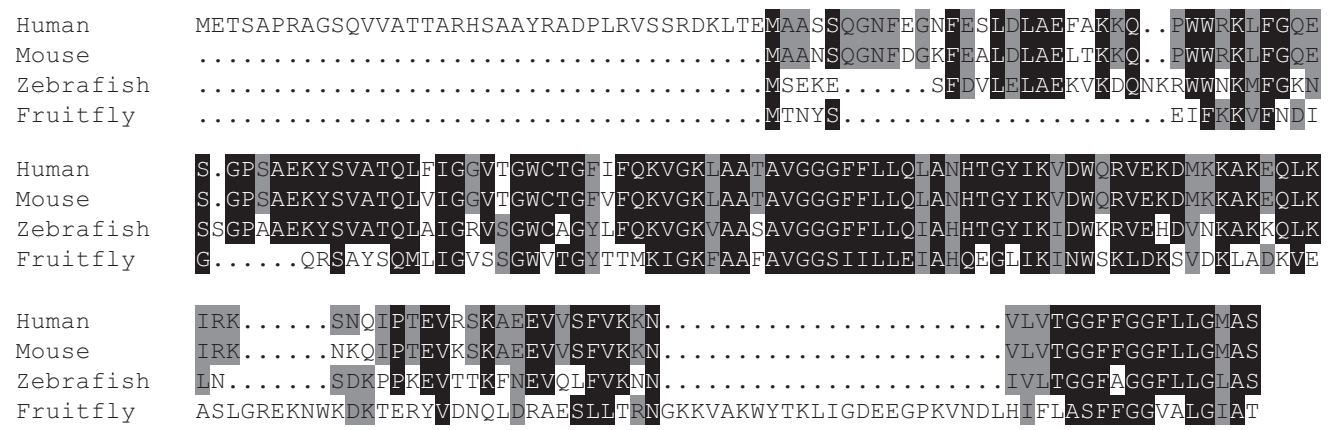

b
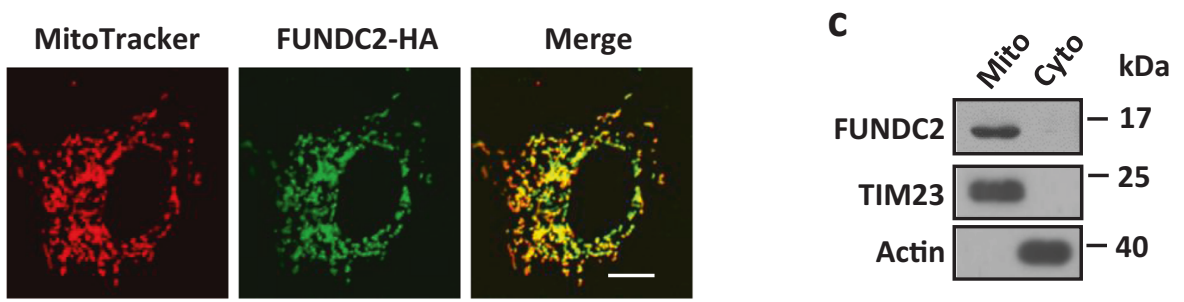

d

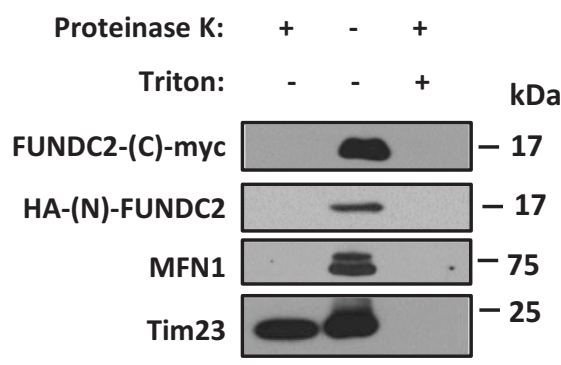

Fig. 1 Identification of FUNDC2 as a mitochondrial protein. a Protein sequence alignment of FUNDC2 from different species using ClustalW. b Mitochondrial localization of FUNDC2. Mouse embryonic fibroblasts (MEFs) expressing HA-tagged FUNDC2 were stained with an HA antibody and MitoTracker. Scale bar, $10 \mu \mathrm{m}$. c Immunoblot

reduced the platelet half-life (from $\sim 61$ to $\sim 42 \mathrm{~h}$ ) in response to hypoxia (Fig. 3a). To further confirm that changes in circulating $t_{1 / 2}$ reflected properties intrinsic to platelets, we performed reciprocal adoptive transfers. Upon transfer into WT recipients, platelets in FUNDC2-null mice were cleared more quickly than those in WT animals under hypoxia stress, with half-lives comparable to those seen in WT mice. Conversely, the clearance of WT platelets transferred into FUNDC2-null mice was identical regardless of the recipient's genotype (Fig. 3b, c). Furthermore, apoptosis markers including a decrease in mitochondrial membrane potential and phosphatidylserine exposure in FUNDC2deficient platelets were also observed by Annexin $\mathrm{V}$ and TMRE staining (Fig. 3d, e). The ex vivo assays also showed that platelets from FUNDC2-null mice were much more sensitive to apoptotic stimulation than WT ones. In addition, this hypoxia-induced apoptosis was remarkably blocked by apoptosis/caspase inhibitor Z-VAD-FMK

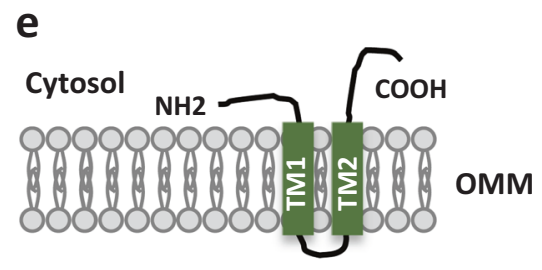

analysis of HeLa cytosolic (Cyto) or mitochondrial (Mito) fractions. d FUNDC2 is localized on the outer mitochondrial membrane. HeLa mitochondria expressing FUNDC2 with C-terminal myc and N-HA were immunoblotted. e Schematic diagram of FUNDC2 topology. TM transmembrane domain, OMM outer mitochondrial membrane

(Fig. 3f). All these findings reflect the protective role of FUNDC2 in platelet maintenance.

\section{FUNDC2 selectively binds PIP3 via its PIP3-binding motif}

In the study of FUNDC2 biological function, bioinformatics analysis predicted the lipid-binding potential of this protein. To verify the physical interaction between FUNDC2 and lipids, membranes spotted with a number of biologically active phospholipids and phosphoinositides were incubated with purified recombinant FUNDC2 protein. The lipid-protein overlay assay showed that FUNDC2 interacted predominantly with PIP3 (Fig. 4a). FUNDC2 was also detected by immunoblotting after immunoprecipitation of endogenous PIP3 from HeLa cells expressing FUNDC2-HA (Fig. 4b). Moreover, affinity pulldown assays using PIP3-coated beads and recombinant FUNDC2 
a
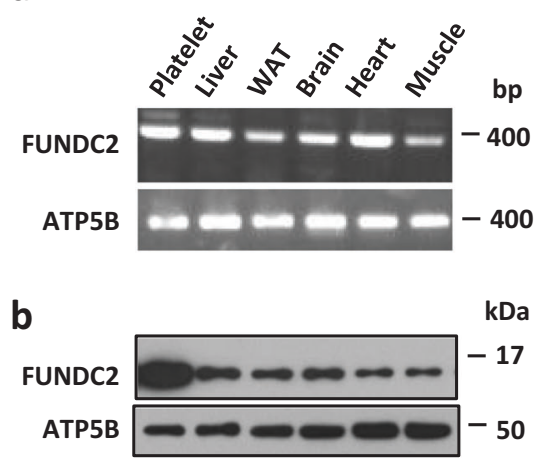

C

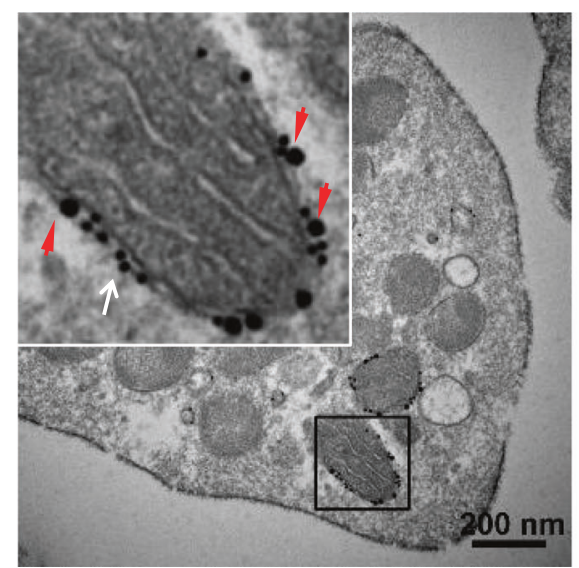

d

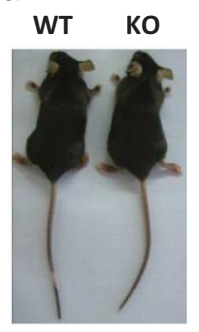

e

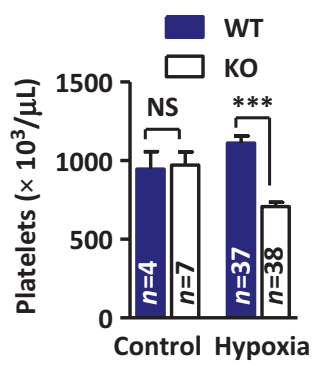

f

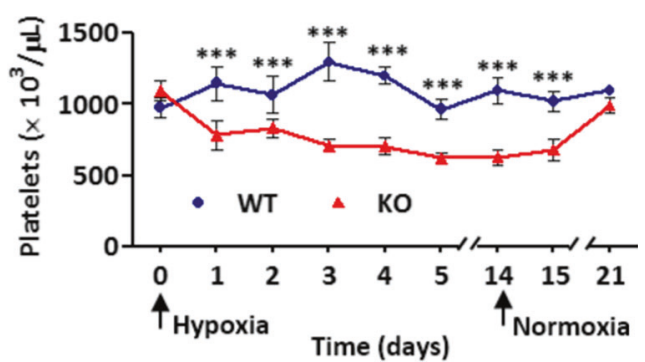

g

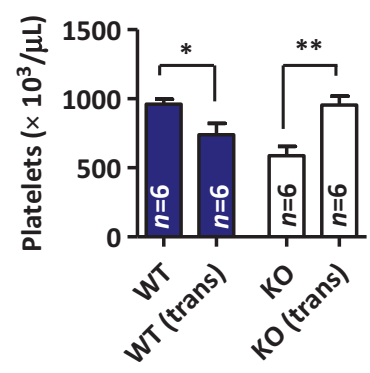

Fig. 2 FUNDC2 knockout mice display hypoxia-induced thrombocytopenia. a The RNA of indicated mouse tissues was extracted and the product of reverse transcription-PCR was shown. b Expression profiling of mouse tissues for FUNDC2. WAT white adipose tissue. Same mitochondrial content in each sample. c Immunogold electron micrograph of FUNDC2 in platelets. Insert: enlarged view of immunogold particles conjugated with antibodies against Nix (mitochondrial outer membrane protein, $10 \mathrm{~nm}$ particles, white arrow) or FUNDC2 (20 nm particles, red arrowheads). d FUNDC2 knockout (KO) mouse and the wild-type (WT) littermate. e Platelet counts were determined from mice treated with hypoxia for 5 days. $f$ Mice were exposed to hypoxia and then put back in normoxia for indicated days and platelet counts were determined ( $n=5-10$ mice). $\mathbf{g}$ Transplantation (trans) of bone marrow. Mice were treated with hypoxia for 5 days $(n=6$ mice) demonstrated the direct binding of FUNDC2 to PIP3 (Fig. 4c). Next, we found that deletion of a unique motif which consists of 11 amino acids abolished the PIP3binding ability of FUNDC2. In contrast, other mutants of FUNDC2 carrying a variety of truncations had similar activities to that of the full-length protein (Fig. 4d). Additionally, overexpression of FUNDC2 in HeLa cells recruited PIP3 to mitochondria. By contrast, the loss-offunction FUNDC2 $\triangle \mathrm{PB}$ can not recruit PIP3 to mitochondria (supplemental Figure S4a, b, and c). Consistent with the role of PIP3 in activating AKT, overexpression of FUNDC2 constitutively elevated the phosphorylation of AKT in mitochondria, but overexpression of the mutant lacking $\mathrm{PB}$ motif (FUNDC2 $\triangle \mathrm{PB}$ ) failed to activate phospho-AKT (supplemental Figure S4d). This PIP3binding (PB) motif at the $\mathrm{N}$ terminus of FUNDC2 is highly conserved from mouse to human (Fig. 4e), and is distinct from pleckstrin-homology $(\mathrm{PH})$ domain [27], which is well-known to bind PIP3. To further define PB motif, a polypeptide encompassing its amino acid residues (KFEALDLAEL) was synthesized. Remarkably, this polypeptide severely impaired the binding of FUNDC2 to PIP3 (Fig. 4f), probably because it competitively interacted with PIP3. In brief, our data indicate that FUNDC2 directly and selectively binds to PIP3 via its PB motif.

\section{FUNDC2 is essential for AKT signaling in a PIP3/ PI3K-dependent manner}

Next, we performed co-immunoprecipitation assays to validate the interaction between endogenous PIP3 and FUNDC2 in resting platelets (Fig. 5a). Moreover, immunostaining assay and ELISA both revealed that PIP3 level of FUNDC2-KO platelets was markedly reduced compared to WT mice (Fig. 5b and supplemental Figure S5). PIP3 is essential for the activation of AKT kinase which regulates cell fate in mammals. Therefore, we hypothesized that FUNDC2 maintains platelet survival via the PIP3/AKT signaling pathway. Then we tested AKT phosphorylation in FUNDC2-null platelets. Markedly, FUNDC2 deficiency severely attenuated AKT phosphorylation. Furthermore, AKT phosphorylation was totally inhibited by hypoxia 
Table 1 Peripheral blood cell values of mice

\begin{tabular}{lllll}
\hline & WT & KO & WT (hypoxia) & KO (hypoxia) \\
\hline Erythrocytes $\left(\times 10^{6} / \mu \mathrm{l}\right)$ & $10.0 \pm 0.2$ & $10.0 \pm 0.1$ & $10.4 \pm 0.5$ & $10.5 \pm 0.3$ \\
Reticulocytes $(\%)$ & $1.0 \pm 0.2$ & $1.4 \pm 0.2$ & $1.1 \pm 0.1$ & $1.5 \pm 0.2$ \\
Leukocytes $\left(\times 10^{3} / \mu \mathrm{l}\right)$ & $8.8 \pm 0.8$ & $9.2 \pm 0.8$ & $2.8 \pm 0.3$ & $2.7 \pm 0.3$ \\
Platelets $\left(\times 10^{3} / \mu \mathrm{l}\right)$ & $946 \pm 111$ & $972 \pm 83$ & $1111 \pm 46$ & $708 \pm 28^{* * *}$ \\
MPV $\left(\times 10^{-9} \mu \mathrm{l}\right)$ & $6.1 \pm 0.1$ & $6.0 \pm 0.1$ & $6.3 \pm 0.1$ & $6.1 \pm 0.1$ \\
$n$ & 4 & 7 & 37 & 38 \\
\hline
\end{tabular}

Data are mean \pm SEM

$M P V$ mean platelet volume.

$* * * p<0.001$ versus WT (Hypoxia, $8 \% \mathrm{O}_{2}$ for 5 days)
Fig. 3 FUNDC2 deficiency shortens platelet lifespan in response to stress. Platelet clearance assay and adoptive platelet transfer assay were carried out as in Methods Section. a Platelet lifespan in mice with the indicated manipulation ( $n=5-10$ mice). $\mathbf{b}$ Biotinylated platelets from donor mice were adoptively transferred into recipients. c Platelet lifespan ( $n=5-10$ mice). d, e Analysis of apoptotic platelets in FUNDC2-deficient mice by TMRE (d) or annexin V (e) staining. f Platelets were isolated from mice and treated ex vivo with $8 \% \mathrm{O}_{2}$ for $4 \mathrm{~h}$ (pretreated with caspase inhibitor, 5 $\mu \mathrm{M}$ Z-VAD-FMK, or not). Each symbol represents an individual animal
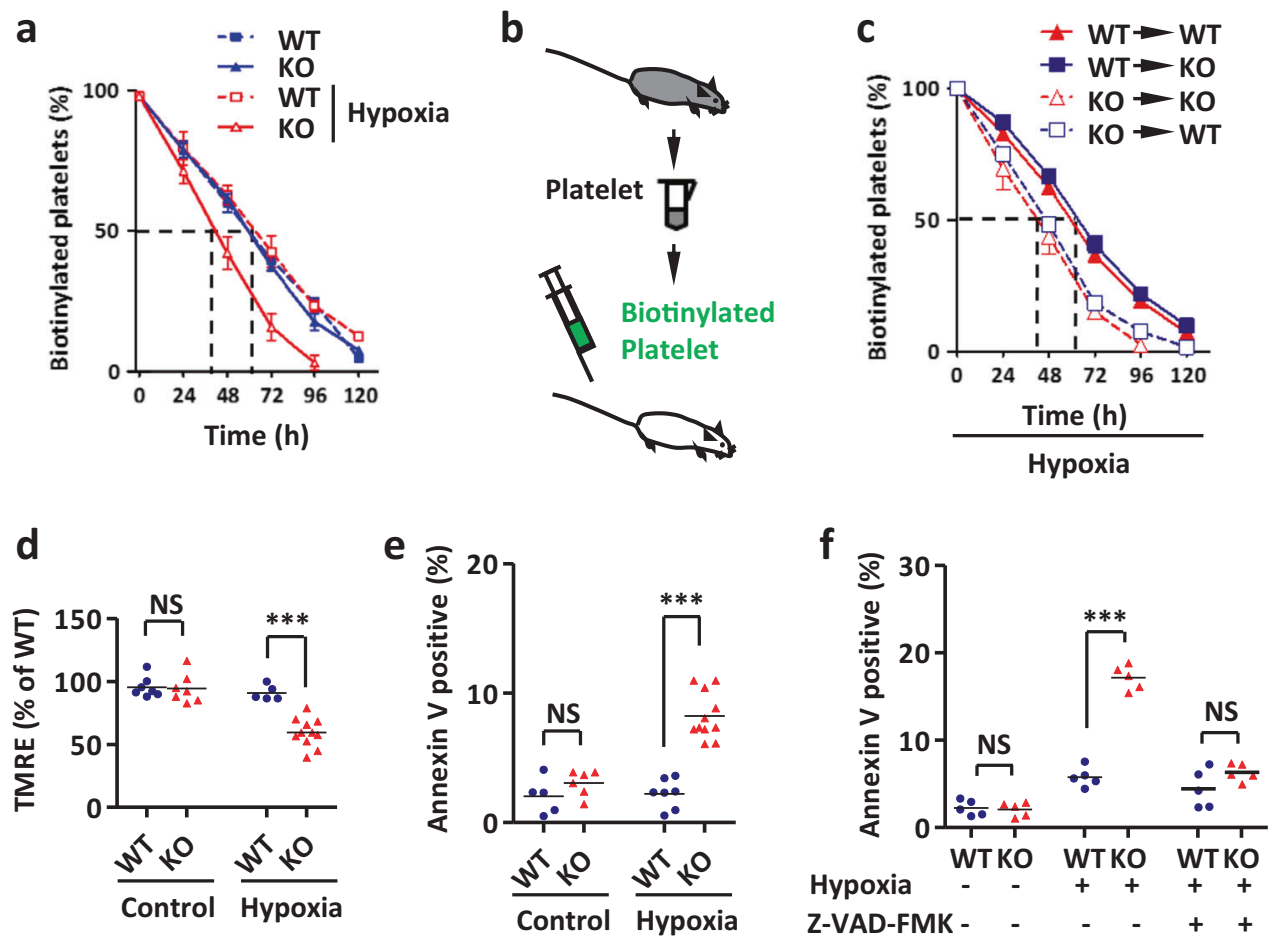

(Fig. 5c, also see Fig. 5e). Consistently, the PIP3independent activation of Src, ERK, or AMPK kinases remained intact in FUNDC2-deficient platelets (supplemental Figure S6), indicating that FUNDC2 mediates AKT activation in a PIP3-dependent manner. PI3K is essential for PIP3/AKT stimulation [20]. Thus, we tested the role of $\mathrm{PI} 3 \mathrm{~K}$ in the FUNDC2/PIP3/AKT pathway. PI3K inhibitor (LY294002) totally blocked AKT phosphorylation in WT or KO platelets (Fig. 5c). Furthermore, LY294002 induced platelet apoptosis in WT mice in response to hypoxia, similar to KO ones (Fig. 5d). The differences of AKT phosphorylation and platelet phenotype between WT and KO mice can be abrogated by LY294002, which suggests that FUNDC2 regulates AKT signaling pathway in a PI3Kand PIP3-dependent manner.

\section{The FUNDC2/AKT/BCL-xL axis protects platelets from apoptosis}

Next, we investigated the regulatory mechanism whereby FUNDC2/AKT maintains platelet survival. In FUNDC2deficient platelets, AKT phosphorylation was suppressed. Consistently, the phosphorylation of BAD, which is a substrate of AKT in mitochondria [27, 28], was also attenuated in the absence of FUNDC2. Furthermore, BAD phosphorylation was completely abrogated by hypoxia stress in $\mathrm{KO}$ platelets (Fig. 5e). The dephosphorylation of BAD enhanced its inhibitory interaction with $\mathrm{BCL}-\mathrm{x}_{\mathrm{L}}$, a pro-survival protein on mitochondrial surface, which protects platelets from apoptosis [14]. Markedly, the amount of $\mathrm{Bcl}-\mathrm{x}_{\mathrm{L}}$ that was coimmunoprecipitated by BAD was increased in FUNDC2- 

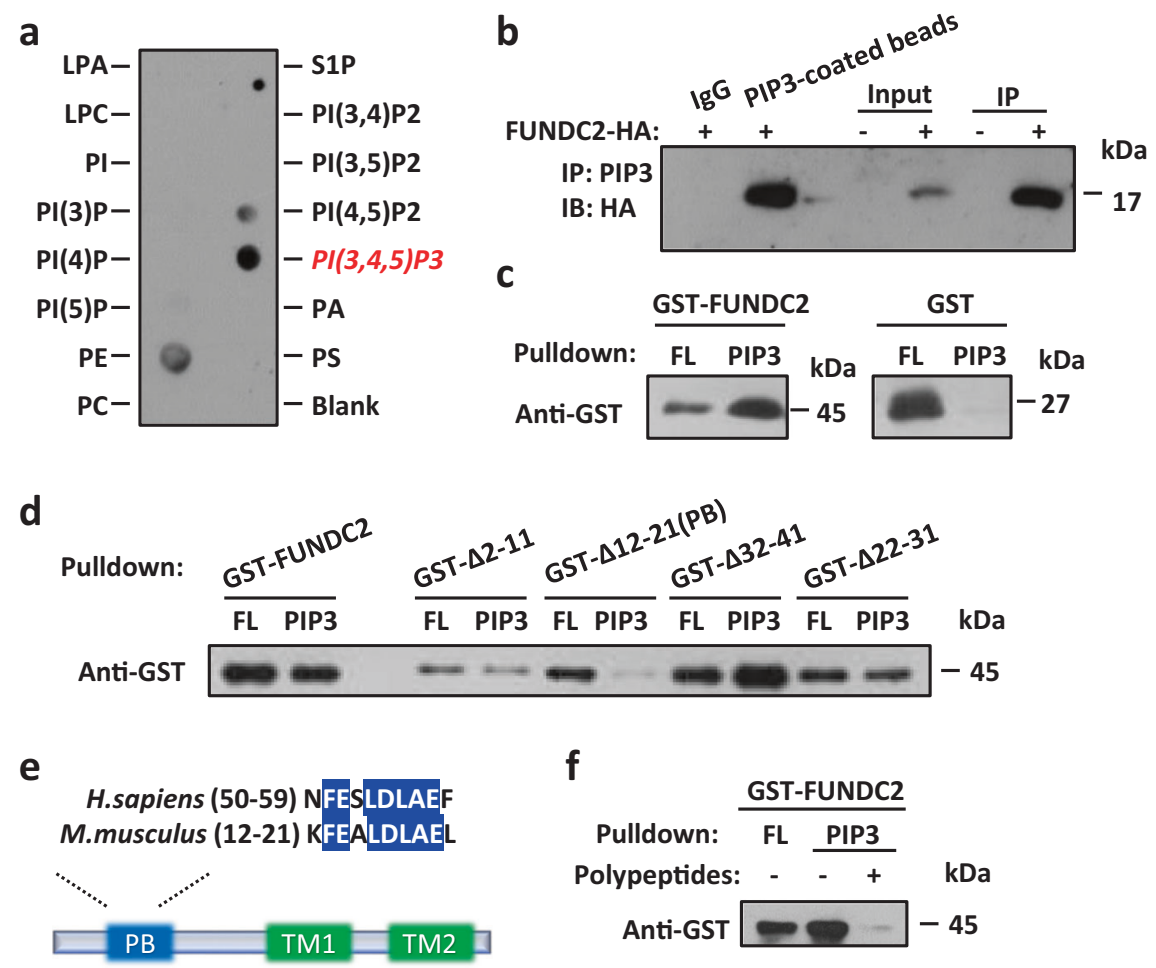

Fig. 4 FUNDC2 is a specific PIP3-binding protein. a Interaction between FUNDC2 and PIP3. LPA lysophosphatidic acid; LPC lysophosphocholine, S1P sphingosine-1-phosphate, PI Phosphatidylinositol, $P A$ phosphatidic acid, $P C$ phosphatidylcholine, $P E$ phosphatidylethanolamine, $P S$ phosphatidylserine. b PIP3 was immunoprecipitated (IP) from HeLa cells expressing FUNDC2-HA and then immunoblotted (IB) for FUNDC2. IgG was used as the negative control and PIP3-coated beads was used as the positive control. c FUNDC2 directly binds PIP3. Pulldown assays were

KO mice than the WT control both in hypoxia and normal conditions (Fig. 5f, and supplemental Figure S7 and S8). Thus, FUNDC2 deficiency caused defects of AKT and BAD phosphorylation, and enhanced $\mathrm{BAD}-\mathrm{BCL}-\mathrm{x}_{\mathrm{L}}$ interaction, which led to BCL- $\mathrm{x}_{\mathrm{L}}$ inhibition. Accordingly, FUNDC2-KO platelets are sensitive to hypoxic stress. Upon the apoptotic stimulation, the inactive $\mathrm{BCL}-\mathrm{x}_{\mathrm{L}}$ failed to prevent downstream apoptotic cascade markers, including a decrease in mitochondrial membrane potential (Fig. 3d), phosphatidylserine exposure (Fig. 3e), and caspase activation (Fig. 5e). Moreover, similar to KO mice, LY294002 also enhanced BAD-BCL- $\mathrm{x}_{\mathrm{L}}$ interaction in WT platelets (supplemental Figure S9), which is consistent that LY294002 inhibited AKT phosphorylation (Fig. 5c) and induced platelet apoptosis (Fig. 5d) of WT mice. It further confirmed that FUNDC2 regulates platelets lifespan in AKT-BAD-BCL- $\mathrm{x}_{\mathrm{L}}$ signaling pathway.

\section{Discussion}

Conclusively, our overall data discovered a novel FUNDC2/ $\mathrm{AKT} / \mathrm{BCL}-\mathrm{x}_{\mathrm{L}}$ axis at mitochondrial surface which protects

performed using PIP3-coated beads with recombinant proteins and analyzed by anti-GST immunoblotting. $F L$ flowthrough. d Identification of the PIP3-binding (PB) motif. Pulldown assays were performed as in (c). e Schematic diagram of FUNDC2. Insert: sequence alignment of motif from human and mouse. TM transmembrane domain. f PIP3coated beads were pre-treated with a synthetic peptide encompassing the residues (KFEALDLAEL) of murine PB motif. Pulldown assays were performed as in $\mathbf{c}$

platelets from apoptosis under stress. FUNDC2 mediates AKT activation in a PIP3- and PI3K-specific manner, which suppresses the co-localized apoptosis machinery of $\mathrm{BAD} /$ $\mathrm{BCL}-\mathrm{x}_{\mathrm{L}}$ in mitochondria and provides survival advantage for platelets. FUNDC2 deficiency severely abrogates AKT phosphorylation and shortens the lifespan of circulating platelets, resulting in thrombocytopenia in response to apoptotic stress such as hypoxia. This newly characterized FUNDC2/AKT/BCL- $x_{L}$ axis in this report signifies a Yin and Yang balance (Fig. 5g) in controlling platelet lifespan at the single organelle level, as well as represents an important paradigm for fate of cells with a high turnover rate.

Mitochondria function as signaling hub in mammalian cells since multiple OMM proteins play as mediators in apoptosis, mitophagy, and innate immunity. Numerous mitochondrial proteins were found to be phosphorylated and much of the work was centered on translocation of kinases onto mitochondria for such effect $[23,29,30]$. Importantly, our data implicate a novel OMM protein in the regulation of mitochondrion-centered AKT signaling, which is dependent of PIP3 and PI3K. Moreover, the FUNDC2's effects on mitochondrial apoptosis and platelet 
a

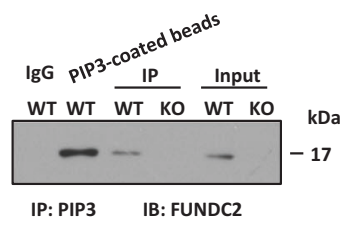

b
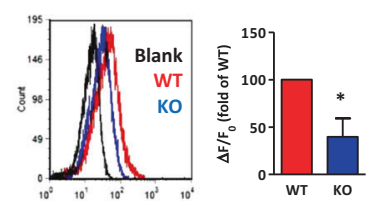

C

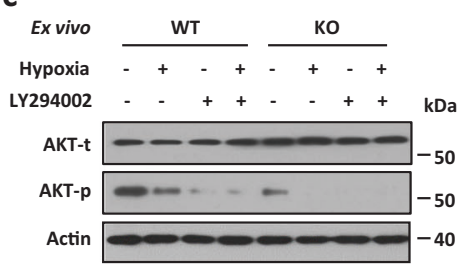

d

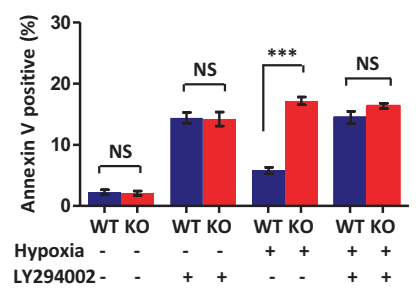

e

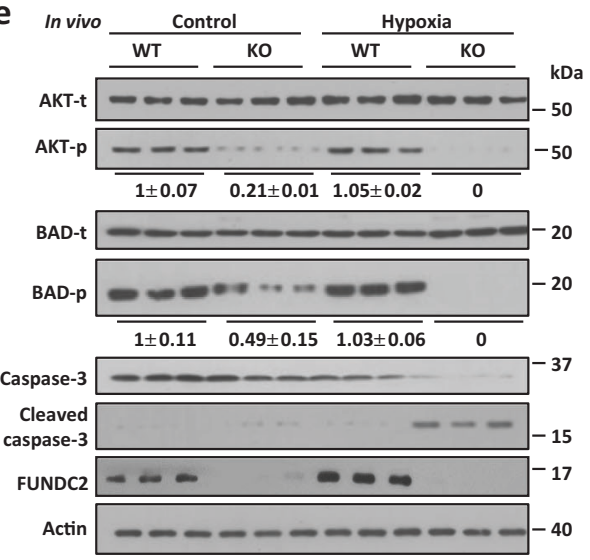

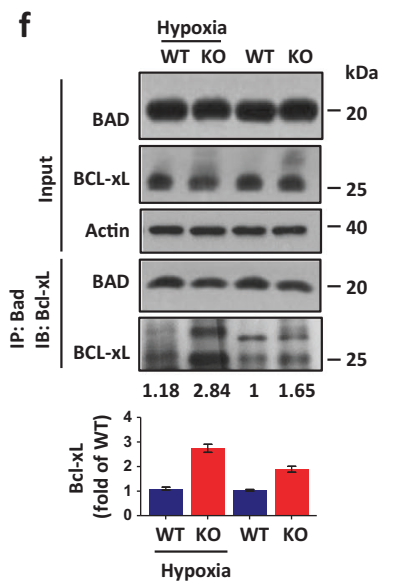

g

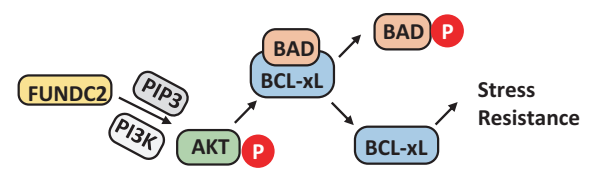

FUNDC2 KO $\rightarrow \underset{\text { AKT }}{\text { PIP3 } \downarrow \text { BCL-xL }} \rightarrow \underset{\text { BCL-xL }}{\text { BAD }} \rightarrow \underset{\text { apoptosis }}{\text { Hypoxia }}$
Fig. 5 FUNDC2 is essential for AKT/BAD/BCL- $\mathrm{x}_{\mathrm{L}}$ axis in platelets. a PIP3 was immunoprecipitated (IP) from resting platelets and immunoblotted (IB) for FUNDC2. IgG negative control, PIP3-coated beads, positive control. b Immunostaining and flow cytometry analysis of PIP3 in platelets isolated from mice in hypoxia $(n=4$ mice). c, $\mathbf{d}$ Platelets were isolated from mice and treated ex vivo with $8 \% \mathrm{O}_{2}$ for 2 h (c) or $4 \mathrm{~h}$ (d) (pre-treated with PI3K inhibitor, $5 \mu \mathrm{M} \mathrm{LY} 294002$, or not). For d, Data are mean \pm SEM $(n=5$ mice). e, f Mice were treated with hypoxia (5 days) and platelets were immunoblotted. At least 3 times of experiments were carried out. The grayscale values of the

counts under hypoxic stress is specific. Our previous data showed that FUNDC1 contains the LC3-interacting region (LIR) motif, which is essential for FUNDC1-mediated mitophagy in platelets [23, 24]. However, FUNDC2 does not contain the LIR motif and no mitophagy can be detected in FUNDC2-KO platelets or in cultured HeLa cells. We also found that FUNDC1 deficiency only affects mitochondrial quality and platelet activity without reducing platelet number under the identical conditions (data not shown). Whether does FUNDC2 affect AKT signaling by interfering with mitochondrial activities? We tested the mitochondrial activities by measuring the respiratory functions in WT and FUNDC2-KO platelets, with the treatment of hypoxia or not. Despite the decrease of the oxygen consumption rate after the treatment of hypoxia, there was no difference in the KO platelets and the WT ones (supplemental Figure S10). It suggests that the deficiency of FUNDC2 has no effects on mitochondrial metabolic functions. Moreover, pull-down assays showed that FUNDC2 have no direct interaction with AKT or PDK1, which is required for AKT phosphorylation (supplemental Figure S11). However, whether AKT can regulate FUNDC2 in a feedback mechanism bands were analyzed with ImageJ software and are presented below the corresponding bands to show the band intensities. For f, representative western blots and averaged data are shown. The interaction between BCL- $\mathrm{x}_{\mathrm{L}}$ and BAD was enhanced in FUNDC2-deficient platelets. Note the augmented BCL- $\mathrm{x}_{\mathrm{L}}$ that was co-immunoprecipitated by BAD in KO mice. $g$ Schematic model showing that FUNDC2 mediates AKT activation in a PIP3-specific manner. This signaling pathway protects platelets against apoptotic stress (hypoxia) by regulating the mitochondrial apoptosis machinery $\left(\mathrm{BAD} / \mathrm{BCL}-\mathrm{x}_{\mathrm{L}}\right)$

needs more research. FUNDC2 can recruit PIP3 to mitochondria using the PIP3-binding motif (supplemental Figure S4). In spite of the mechanism, FUNDC2 possibly plays as an anchor and mediates the transfer of PIP3 to mitochondria, and the binding of FUNDC2-PIP3 stabilize PIP3 levels, which is required for AKT phosphorylation and BAD-BCL- $\mathrm{x}_{\mathrm{L}}$ signaling.

Platelets in peripheral blood are exposed to a diversity of stresses (e.g., shear force, high-oxygen, oxidative damage, and hypoxia) and undergo apoptosis in response to these stimuli $[4,15,18,31-36]$. Hypoxia is a major pathological mechanism that underlies atherosclerosis, myocardial infarction, acute coronary syndromes, and ischemia/reperfusion (I/R) injury. Platelets also play essential role in these cardiovascular diseases [37-39]. Thus, we used chronic and mild hypoxia as a pathological condition to treat mice. Some reports showed that early-onset thrombocytopenia was associated with placental insufficiency and hypoxia in preterm neonates, which was suggested to be the result of decreased platelet production [36]. Long-term hypoxia ( $\sim 6 \% \mathrm{O}_{2}$ for 10 days) was thought to decrease platelet counts in mice, which was linked to the inhibition of 
production of thrombopoietin (TPO) [31, 32, 34, 35]. However, previous studies of different groups have demonstrated that hypoxia of short duration $\left(\sim 8 \% \mathrm{O}_{2}\right.$ for 1-3 days) increased platelet counts [4, 33, 35], which is consistent with our data that hypoxia ( $\sim 8 \% \mathrm{O}_{2}$ for $1-4$ days) increased platelets in WT mice. And we also showed that TPO is little changed during hypoxia. The mechanism by which short-term hypoxia increased platelets is still unknown. It was suggested that megakaryocytes possibly over-compensatively shed platelets into the circulation in response to hypoxia stress [4]. FUNDC2 mRNA is ubiquitous in a diversity of tissues of mice but the protein level is specifically high in platelets. The possible reason is that FUNDC2 might be highly expressed for platelet maintenance under these apoptotic stimulations. Also, platelets are anuclear and have a high turnover rate. All these may requires accurate modulation of intrinsic apoptosis specifically in a FUNDC2-dependent manner.

BCL-2 family proteins have been established for their critical role in regulating platelet lifespan. The pro-apoptotic protein $\mathrm{BAK}$ and anti-apoptotic $\mathrm{BCL}-\mathrm{x}_{\mathrm{L}}$ perform antagonistic effects on intrinsic apoptosis of platelets [15]. BID and BIM, two of the pro-apoptotic BH3-only proteins, are dispensable for platelet apoptosis [40]. However, our present work showed that another BH3-only protein BAD regulates platelet survival by binding to $\mathrm{BCL}-\mathrm{x}_{\mathrm{L}}$, which is consistent with the phenotype that $\mathrm{bad}^{-1-}$ mice exhibit thrombocytosis due to prolonged platelet life-span [41]. Thus, our findings extend the role of BCL-2 family proteins in platelet survival. FUNDC2 regulates platelet number in response to hypoxia via $B C L-x_{L}-d e p e n d e n t$ apoptosis. Our data is consistent with the findings that pharmacologic inhibition of BCL- $\mathrm{x}_{\mathrm{L}}$ by ABT737 or ABT263, both the pro-apoptotic BCL-2 homology 3 (BH3) mimetic [17], also leads to dose-dependent thrombocytopenia [15, 18, 42]. Notably, BCL- $\mathrm{x}_{\mathrm{L}}$ inhibition not only reduced platelet count but also largely impaired platelet functions and activation [18, 42]. In our study, FUNDC2 regulates platelet apoptosis via the modulation of BCL- $\mathrm{x}_{\mathrm{L}}$ activity. Thus, it is interesting to evaluate whether FUNDC2 regulates hemostatic function of platelets in bleeding as well as in cardiovascular diseases in further studies. As platelets play essential role for blood hemostasis, wound healing, cardiovascular disease, and immunity, our study paves the new avenue for the regulation of platelet survival at the level of mitochondria and hold promises for fighting these diseases.

Acknowledgements QC was supported by grants NSFC 81130045 and 31520103904. Q.M. was supported by grant NSFC 31500672.

Authors contributions $\mathrm{QM}$ and $\mathrm{QC}$ designed the experiments. QM, CZ, WZ, RZ, NT, DF, and LL carried out the experiments. QM, CZ, and $\mathrm{WZ}$ analyzed and organized the data. QM, HC, JL, and QC wrote the manuscript. All authors discussed the results and commented on the manuscript. QM and CZ contributed equally to this study.

\section{Compliance with ethical standards}

Conflict of interest The authors declare that they have no conflict of interest.

\section{References}

1. Holmsen $\mathrm{H}$. Physiological functions of platelets. Ann Med. 1989;21:23-30.

2. Lee SH, Du J, Stitham J, Atteya G, Lee S, Xiang Y, et al. Inducing mitophagy in diabetic platelets protects against severe oxidative stress. EMBO Mol Med. 2016;8:779-95.

3. Tyagi T, Ahmad S, Gupta N, Sahu A, Ahmad Y, Nair V, et al. Altered expression of platelet proteins and calpain activity mediate hypoxia-induced prothrombotic phenotype. Blood. 2014;123:1250-60.

4. McDonald TP, Cottrell M, Clift R. Effects of short-term hypoxia on platelet counts of mice. Blood. 1978;51:165-75.

5. Delaney MK, Liu J, Kim K, Shen B, Stojanovic-Terpo A, Zheng $\mathrm{Y}$, et al. Agonist-induced platelet procoagulant activity requires shear and a Rac1-dependent signaling mechanism. Blood. 2014;124:1957-67.

6. Begonja AJ, Gambaryan S, Geiger J, Aktas B, Pozgajova M, Nieswandt B, et al. Platelet NAD(P)H-oxidase-generated ROS production regulates alphallbbeta3-integrin activation independent of the NO/cGMP pathway. Blood. 2005;106:2757-60.

7. Bertino AM, Qi XQ, Li J, Xia Y, Kuter DJ. Apoptotic markers are increased in platelets stored at 37 degrees C. Transfusion. 2003;43:857-66.

8. Perrotta PL, Perrotta CL, Snyder EL. Apoptotic activity in stored human platelets. Transfusion. 2003;43:526-35.

9. Li J, Xia Y, Bertino AM, Coburn JP, Kuter DJ. The mechanism of apoptosis in human platelets during storage. Transfusion. 2000;40:1320-9.

10. Vanags DM, Orrenius S, Aguilar-Santelises M. Alterations in Bcl$2 /$ Bax protein levels in platelets form part of an ionomycininduced process that resembles apoptosis. $\mathrm{Br} \mathrm{J}$ Haematol. 1997;99:824-31.

11. Pereira J, Soto M, Palomo I, Ocqueteau M, Coetzee LM, Astudillo $\mathrm{S}$, et al. Platelet aging in vivo is associated with activation of apoptotic pathways: Studies in a model of suppressed thrombopoiesis in dogs. Thromb Haemost. 2002;87:905-9.

12. Ault KA, Knowles C. In vivo biotinylation demonstrates that reticulated platelets are the youngest platelets in circulation. Exp Hematol. 1995;23:996-1001.

13. Kile BT. The role of the intrinsic apoptosis pathway in platelet life and death. J Thromb Haemost. 2009;7(Suppl 1):214-7.

14. Boise LH, Gonzalez-Garcia M, Postema CE, Ding L, Lindsten T, Turka LA, et al. bcl-x, a bcl-2-related gene that functions as a dominant regulator of apoptotic cell death. Cell. 1993;74: 597-608.

15. Mason KD, Carpinelli MR, Fletcher JI, Collinge JE, Hilton AA, Ellis S, et al. Programmed anuclear cell death delimits platelet life span. Cell. 2007;128:1173-86.

16. Kodama T, Takehara T, Hikita H, Shimizu S, Li W, Miyagi T, et al. Thrombocytopenia exacerbates cholestasis-induced liver fibrosis in mice. Gastroenterology. 2010;138:2487-98. 2498 e2481-2487

17. Oltersdorf T, Elmore SW, Shoemaker AR, Armstrong RC, Augeri DJ, Belli BA, et al. An inhibitor of Bcl-2 family proteins induces regression of solid tumours. Nature. 2005;435:677-81.

18. Schoenwaelder SM, Jarman KE, Gardiner EE, Hua M, Qiao J, White MJ, et al. Bcl-xL-inhibitory BH3 mimetics can induce a transient thrombocytopathy that undermines the hemostatic function of platelets. Blood. 2011;118:1663-74. 
19. Manning BD, Cantley LC. AKT/PKB signaling: navigating downstream. Cell. 2007;129:1261-74.

20. Engelman JA, Luo J, Cantley LC. The evolution of phosphatidylinositol 3-kinases as regulators of growth and metabolism. Nat Rev Genet. 2006;7:606-19.

21. Stokoe D, Stephens LR, Copeland T, Gaffney PR, Reese CB, Painter GF, et al. Dual role of phosphatidylinositol-3,4,5-trisphosphate in the activation of protein kinase B. Science. 1997;277:567-70.

22. Bijur GN, Jope RS. Rapid accumulation of Akt in mitochondria following phosphatidylinositol 3-kinase activation. J Neurochem. 2003;87:1427-35.

23. Liu L, Feng D, Chen G, Chen M, Zheng Q, Song P, et al. Mitochondrial outer-membrane protein FUNDC1 mediates hypoxia-induced mitophagy in mammalian cells. Nat Cell Biol. 2012;14:177-85.

24. Zhang W, Ren H, Xu C, Zhu C, Wu H, Liu D, et al. Hypoxic mitophagy regulates mitochondrial quality and platelet activation and determines severity of $\mathrm{I} / \mathrm{R}$ heart injury. Elife 2016;5: 21407-27.

25. Sheen CR, Jewell UR, Morris CM, Brennan SO, Ferec C, George $\mathrm{PM}$, et al. Double complex mutations involving F8 and FUNDC2 caused by distinct break-induced replication. Hum Mutat. 2007;28:1198-206.

26. Li K, Wang L, Cheng J, Zhang L, Duan H, Lu Y, et al. [Screening and cloning gene of hepatocyte protein interacting with hepatitis $\mathrm{C}$ virus core protein]. Zhonghua Shi Yan He Lin Chuang Bing Du Xue Za Zhi. 2002;16:351-3.

27. DiNitto JP, Cronin TC, Lambright DG. Membrane recognition and targeting by lipid-binding domains. Sci STKE. 2003;2003: re16.

28. Datta SR, Dudek H, Tao X, Masters S, Fu H, Gotoh Y, et al. Akt phosphorylation of BAD couples survival signals to the cellintrinsic death machinery. Cell. 1997;91:231-41.

29. Cloonan SM, Choi AM. Mitochondria: sensors and mediators of innate immune receptor signaling. Curr Opin Microbiol. 2013;16:327-38.
30. McBride HM, Neuspiel M, Wasiak S. Mitochondria: more than just a powerhouse. Curr Biol. 2006;16:R551-560.

31. Birks JW, Klassen LW, Gurney CW. Hypoxia-induced thrombocytopenia in mice. J Lab Clin Med. 1975;86:230-8.

32. Shreiner DP, Levin J. The effects of hemorrhage, hypoxia, and a preparation of erythropoietin on thrombopoiesis. J Lab Clin Med. 1976;88:930-40.

33. Jackson CW, Edwards CC. Biphasic thrombopoietic response to severe hypobaric hypoxia. Br J Haematol. 1977;35:233-44.

34. Langdon JR, McDonald TP. Effects of chronic hypoxia on platelet production in mice. Exp Hematol. 1977;5:191-8.

35. McDonald TP. Platelet production in hypoxic and RBC-transfused mice. Scand J Haematol. 1978;20:213-20.

36. Saxonhouse MA, Rimsza LM, Stevens G, Jouei N, Christensen $\mathrm{RD}$, Sola MC. Effects of hypoxia on megakaryocyte progenitors obtained from the umbilical cord blood of term and preterm neonates. Biol Neonate. 2006;89:104-8.

37. Nagareddy P, Smyth SS. Inflammation and thrombosis in cardiovascular disease. Curr Opin Hematol. 2013;20:457-63.

38. Gawaz M. Role of platelets in coronary thrombosis and reperfusion of ischemic myocardium. Cardiovasc Res. 2004;61:498-511.

39. Ruggeri ZM. Platelets in atherothrombosis. Nat Med. 2002;8:1227-34.

40. Kodama T, Takehara T, Hikita H, Shimizu S, Shigekawa M, Li $\mathrm{W}$, et al. BH3-only activator proteins Bid and Bim are dispensable for Bak/Bax-dependent thrombocyte apoptosis induced by Bcl-xL deficiency: molecular requisites for the mitochondrial pathway to apoptosis in platelets. J Biol Chem. 2011;286:13905-13.

41. Kelly PN, White MJ, Goschnick MW, Fairfax KA, Tarlinton DM, Kinkel SA, et al. Individual and overlapping roles of BH3-only proteins Bim and Bad in apoptosis of lymphocytes and platelets and in suppression of thymic lymphoma development. Cell Death Differ. 2010;17:1655-64.

42. Vogler M, Hamali HA, Sun XM, Bampton ET, Dinsdale D, Snowden RT, et al. BCL2/BCL-X(L) inhibition induces apoptosis, disrupts cellular calcium homeostasis, and prevents platelet activation. Blood. 2011;117:7145-54. 